\title{
Central Control System of Broadcast Network Based on AoIP Technology
}

\author{
Jian $\mathrm{He}^{1}$ and Zhao Zhang ${ }^{2}$ \\ School of Automation Engineering, University of Electronic Science and \\ Technology of China, Chengdu, China \\ hejian@uestc.edu.cn ${ }^{1}$,yxzhangzh@163.com ${ }^{2}$
}

\begin{abstract}
The digital broadcast network system can meet different business needs of broadcast station at all levels. Its central control system is the key component for the signal transmission and exchange in the whole system as well as between the system and the external system. The central control system is the signal center of the whole radio link. And it integrates with each independent technical system of the whole station to realize the sharing of the signal resource. For the adaptation to technical progress of broadcast network system, this paper presents a new generation of full digital, networked central control system which is based on the latest AoIP digital audio transmission technology. This paper also puts forward the design scheme of the system and its technical features based on the demand analysis.
\end{abstract}

Keywords: AoIP technology, central control system, broadcast network

\section{Introduction}

\subsection{The Structure of Digital Broadcast Network System}

The digital broadcast network system consists of program producing system, business management system, broadcasting system, news manuscript system, digital director system, medium resource system, intelligent control system and so on. The system meets different business needs of broadcast station at all levels.

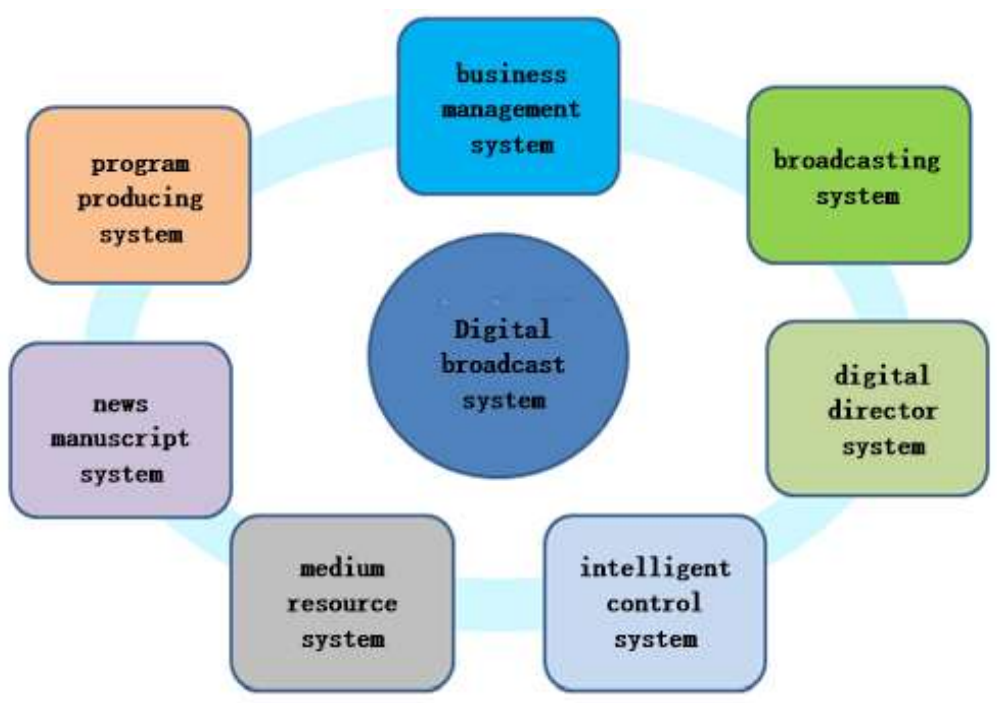

Figure 1. The Structure of Digital Broadcast Network System 
The central control system is the key component for the signal transmission and exchange in the whole system as well as between the system and the external system. This system also takes on the tasks of monitoring, adjusting, processing and scheduling of all kinds of the broadcasting audio signals. At the same time, it is responsible for the whole station's clock signal system. The central control system is the signal center of the whole radio link, and it integrates with each independent technical system of the whole station to realize the sharing of the signal resource.

The central control system has the following functions.

(1) Switch and distribute of the program, and send continuous high quality digital signals to the transmitting station and cable broadcast;

(2) Receive and forward normal signals and live signals, and switch and route automatically or manually;

(3) Responsible for sending the audio source signals to each of the live studios and recording studios, and sending the signals back to the live studios and recording studio by matrix signals;

(4) Real-timely monitor and slowly record (with a slow recording station) the program's multi points (live, pre-launch, air receiver);

(5) When the fault occurs, it has sound and light alarm, switching emergency intelligently and recording;

(6) Provide standard time, six rings, hourly chime signal (through the network calibration time, broadcast by automatic timekeeping workstation) for each broadcast station and each network;

(7) Video monitoring functions; Transmit through the main channel; and the inputs of the device are audio digital signals;

(8) Realize digital transmission, intelligent switch, network management.

Broadcast network system's program production, broadcast, business management, news, media, and other systems have been mature products, which have been used in radio stations at all levels. Due to limited space, the details are not introduced here.

\subsection{The Limitation of the Traditional Central Control System}

The traditional central control system is an application system which is based on analog or digital devices as the object and is based on switched signal as the core component. Its core equipment of audio matrix is redundancy architecture and all audio signals are high-density integrated to the audio matrix. Leading that radio digital network's construction and operation with central control system in signal format, transmission interface, data exchange phase separation is not really connected, and unable to realize signals' interconnection in the level of network and data. Adding a large scale of $\mathrm{DA} / \mathrm{AD}$ conversion and audio distribution equipment not only reduces the signal technical indicators also increases the number of failure points. And what the most critical is that the inability can achieve program signal stream's transmission and switching. There are many problems throughout the system. The whole system exists many devices; The signals have the problems of attenuation, distortion, poor anti-interference, and close transmission distance; Core equipment is high density focused. So once it fails, wide influence would suffer. There are a large scale of DA/AD conversion and audio distribution equipment, so the link is complex and the fault points are increasing; the equipment management is complex, and the signal monitoring points are limited; cables are too many and complex to arrangement, so it is difficult to maintain; and it costs too much to construct. Once traditional central control system breaks, it cannot quickly determine the point of failure and recover the broadcast. 


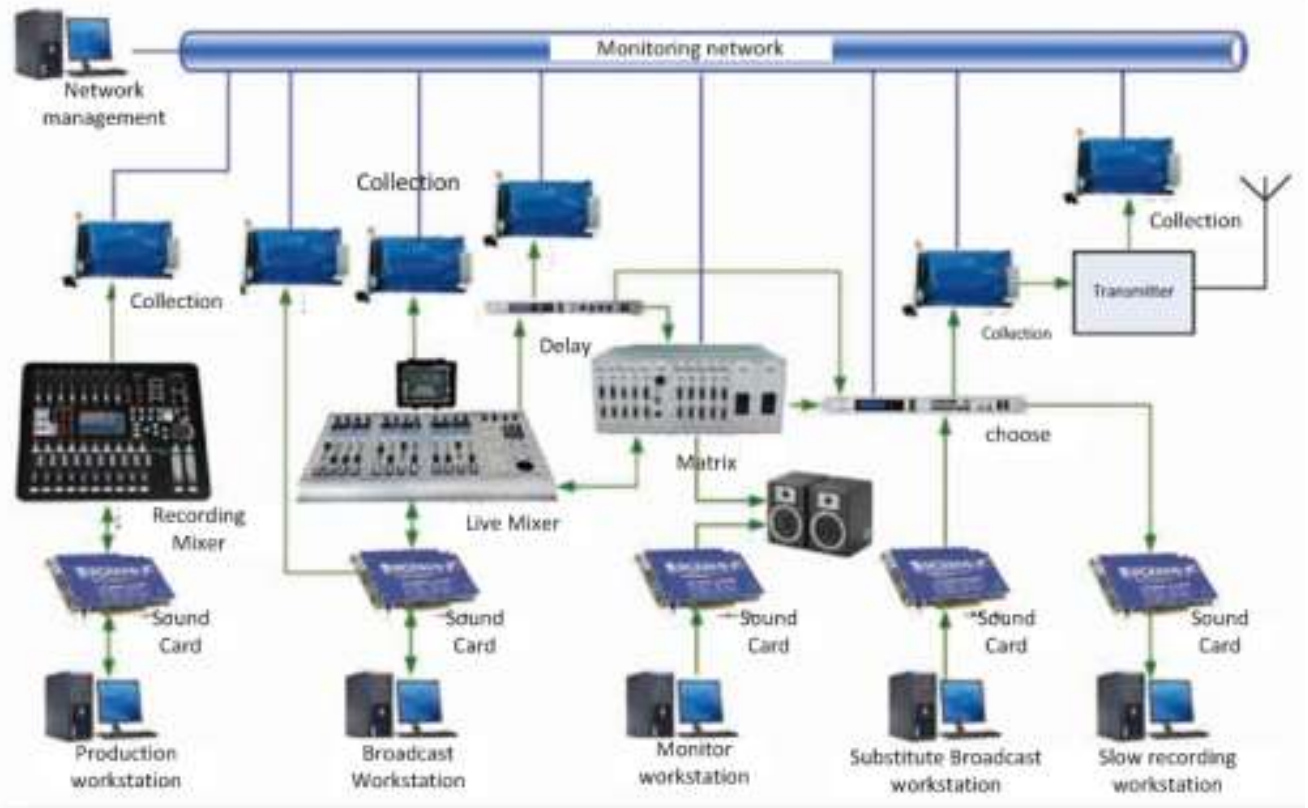

Figure 2. Transmission and Monitoring System of Traditional Sense

\section{Design New Central Control System of Broadcast Network}

For the adaptation to technical progress of broadcast network system, we use the latest AoIP digital audio transmission technology to build a new generation of full digital, networked central control system, which effectively overcomes the inherent limitations in the traditional radio control system, and realizes the transmission and exchange of the network audio signal flow. Compared with the traditional control system, it has obvious advantages.

\subsection{Demand Analysis of the New Generation Central Control System of Broadcast Network}

The new generation of intelligent central control system should be a structure of multilink complementary with dual-routes that can run at the same time. It is a complete digital, networked, intelligent central control system which forms from the living room to the central control to the transmission link. Digital Intelligent Central Control System uses the most cutting-edge technology and the latest broadcast technology products to meet high-tech standards. The basic technology platform is based on digital audio technology, advanced network technology at the whole process. It can deal with faults manually or automatically. All active devices are redundant dual power. The key link devices use redundant dual Ethernet ports, and the devices have a capability of power-down and pass-through.

Broadcasting System's basic framework is that using AoIP technology to realize link from production to broadcast to the total control to the network intelligence management system to digital audio network of work station system;

To meet the requirements of multi-function, multi-means testing, surveillance and monitoring, the system has fault diagnosis functions;

Live Studio system uses AoIP interfaced with digital, network-based, modular, dual power supplies, line plug-in digital mixer; It meets the needs of radio automation and intelligence;

Recording system uses digital equipment to meet the needs of various types of program recording, and it can achieve program staff clipping programs, scheduling programs and 
can have a truly seamless interoperability gapless resource sharing with the central control, broadcast system in the digital audio network transmission.

Using AoIP virtual sound card technology can make slow record, emergency broadcast system from the shackles of professional sound card and make it easier to signal route, which expands the audio production network platform;

Perfect synchronization mechanism makes different sample rate audio signals can be reliably and orderly transferred in the same network;

System configuration is safe and reliable, and the device has no single point of collapse. Its major equipment is redundancy, and it uses warm backup;

In order to improve the reliability of safe broadcasting, the frequency network control device has been adopted independent chassis, which can effectively reduce the correlation between frequency and the mutual influence between each other; In the central control wiring, the frequency of input and output channels are distributed in different input and output boards, so that the effects of the two signals are reduced;

Maintenance is simple and convenient, with automatic and manual fault processing strategy. There is a design principle that when system upgrades and maintenances, it can reach 0 seconds to stop broadcast, 0 risks to upgrade.

\subsection{Introduction of AoIP Digital Audio Transmission Technology}

AoIP digital audio transmission technology is proposed by Audinate in 2003, based on the Ethernet layer 3 high fidelity real-timely multi-channel audio transmission technology, which can transmit uncompressed audio signals at speed up to $512 \mathrm{ch}+512 \mathrm{ch}$, quality at 48KHZ, 24bit through the Ethernet. Due to the adoption of IEEE1588 precision clock transmission protocol, it gets the low-latency jitter of multi-channel audio transmission in the general Ethernet. This technology is regarded as the core technology of the next generation of digital audio, and it provides a low-latency, high-precision and low-cost solution for the audio connection (which can transmit and complicatedly route precision clock signals and professional audio signals in Ethernet [100M or $1000 \mathrm{M}]$ ). Compared with the previous conventional audio transmission technology, it inherits all the advantages of CobraNet and Ether Sound, such as uncompressed digital audio signal can ensure a good sound quality; It can solve the traditional audio transmission in complex wiring problems, and reduce costs; It can adapt to the existing network, without making special configuration; Network audio signal has been labeled in the form of labels. At the same time, it has its own unique advantages as follows.

It has a smaller delay. In the $100 \mathrm{M}$ network bandwidth, when total transmission of audio channels is 3 , the delay is only $34 \mathrm{US}$;

The system automatically adjusts to the available network bandwidth and reduces the time delay to a minimum;

Using the IEEE1588 Precision Time Protocol for clock synchronization;

Using Zeroconf (Zero Configuration Networking) protocol, and using the automatic Configuration server to automatically check the interface device, identification tags and distinguish IP address. It doesn't need to start the top level of the DNS and DHCP services, at the same time it can save the complex manual network configuration;

High compatibility features of the network. Audio signal, control data and other data can flow with sharing the same network;

Self-healing system. In order to avoid the accident that leading to the suspension of audio transmission, the system can set up multiple self-healing mechanisms, such as the clock missing, network failure, etc.

Audio channel transmission mode can be unicast or multicast;

Transferring density can get up to $64 \mathrm{ch}$ in and $64 \mathrm{ch}$ out in $100 \mathrm{M}$ Ethernet, $512 \mathrm{ch}$ in and $512 \mathrm{ch}$ out in $1000 \mathrm{M}$ Ethernet. Use multicast technology can realize software distribution and soft switching in network signals, and audio exchange with PC can also skip the traditional sound card directly by the network implementation; 
Table 1. The Comparison among CobraNet, Ethersound and AoIP

\begin{tabular}{|c|c|c|c|}
\hline Characteristic & CobraNet & Ethersound & AoIP \\
\hline $\begin{array}{l}\text { Fully support } \\
\text { Ethernet }\end{array}$ & Yes & Radio only & Yes \\
\hline $\begin{array}{c}\text { Support the } \\
\text { standard TCP/IP }\end{array}$ & No & No & Yes \\
\hline $\begin{array}{c}\text { Technology AES67 } \\
-2013\end{array}$ & No & No & Yes \\
\hline Sampling rate & $48 \mathrm{~K} / 96 \mathrm{~K}$ & $\begin{array}{c}44.1 \mathrm{~K} / 48 \mathrm{~K} / \\
88.2 \mathrm{~K} / 96 \mathrm{~K} 192 \mathrm{~K}\end{array}$ & $\begin{array}{c}44.1 \mathrm{~K} / 48 \mathrm{~K} / \\
88.2 \mathrm{~K} / 96 \mathrm{~K} 192 \mathrm{~K}\end{array}$ \\
\hline Quantitative depth & 16/20/24bit & 16/20/24bit & 16/20/24bit \\
\hline Jitter time & 40/60ns & $<1 \mathrm{~ns}$ & $<1 \mathrm{~ns}$ \\
\hline $\begin{array}{c}\text { Network } \\
\text { bandwidth } \\
\text { requirements }\end{array}$ & $\begin{array}{l}\text { Do not need to use a } \\
\text { dedicated } 100 \mathrm{M} \\
\text { Ethernet, but Peak } \\
\text { Audio suggest using } \\
\text { gigabit virtual } \\
\text { network (VLAN) }\end{array}$ & $\begin{array}{l}\text { Use a dedicated 100M } \\
\text { Ethernet, or use } \\
\text { gigabit virtual } \\
\text { network (VLAN) }\end{array}$ & $\begin{array}{l}\text { No need to use } \\
\text { special Ethernet }\end{array}$ \\
\hline $\begin{array}{l}\text { The complexity of } \\
\text { design and } \\
\text { maintenance of } \\
\text { network }\end{array}$ & High & Middle & Low \\
\hline $\begin{array}{l}\text { Multiple multicast } \\
\text { mode }\end{array}$ & No & No & Yes \\
\hline $\begin{array}{l}\text { Efficient unicast } \\
\text { mode }\end{array}$ & Partly & No & Yes \\
\hline Audio delay & $1.33 \mathrm{~ms}$ & $125 \mu \mathrm{s}+$ & $34 \mu \mathrm{s}$ \\
\hline $\begin{array}{l}\text { Automatic } \\
\text { optimization of } \\
\text { audio delay }\end{array}$ & No & No & Yes \\
\hline $\begin{array}{l}\text { Same network } \\
\text { using different } \\
\text { speed devices }\end{array}$ & No & No & Yes \\
\hline $\begin{array}{l}\text { Different sampling } \\
\text { frequency is used } \\
\text { for the same } \\
\text { network }\end{array}$ & No & No & Yes \\
\hline $\begin{array}{c}\text { Zero-Conf } \\
\text { Network } \\
\text { configuration }\end{array}$ & No & No & Yes \\
\hline $\begin{array}{l}\text { Technology release } \\
\text { time }\end{array}$ & 1996 & 2002 & 2003 \\
\hline $\begin{array}{c}\text { The maximum } \\
\text { number of devices } \\
\text { that can be } \\
\text { connected to a } \\
\text { network }\end{array}$ & 164 & 65534 & Unlimited \\
\hline $\begin{array}{c}\text { Technology } \\
\text { development / } \\
\text { holder }\end{array}$ & PeakAudio & Digigram & Audinate \\
\hline
\end{tabular}

At present, the mainstream of AoIP technology is Dante and AES67-2013, the former 
has higher market share and maturity, the latter is the international unified standard AES group released in 2013.

\subsection{Design a New Central Control System based on AOIP Technology}

The new generation central control management platform based on AOIP consists of server, terminal equipment, display terminals, the management station. Terminal equipment includes all non AoIP and AOIP audio equipment, as well as video surveillance, computer room environment monitoring and other non-audio equipment, and all this equipment constitute a complete and unified monitoring and control system through redundant IP networks. Running in the terminal and workstation, the management software achieves the various management functions, meets the requirements of the central control system under different security conditions, and different topologies.

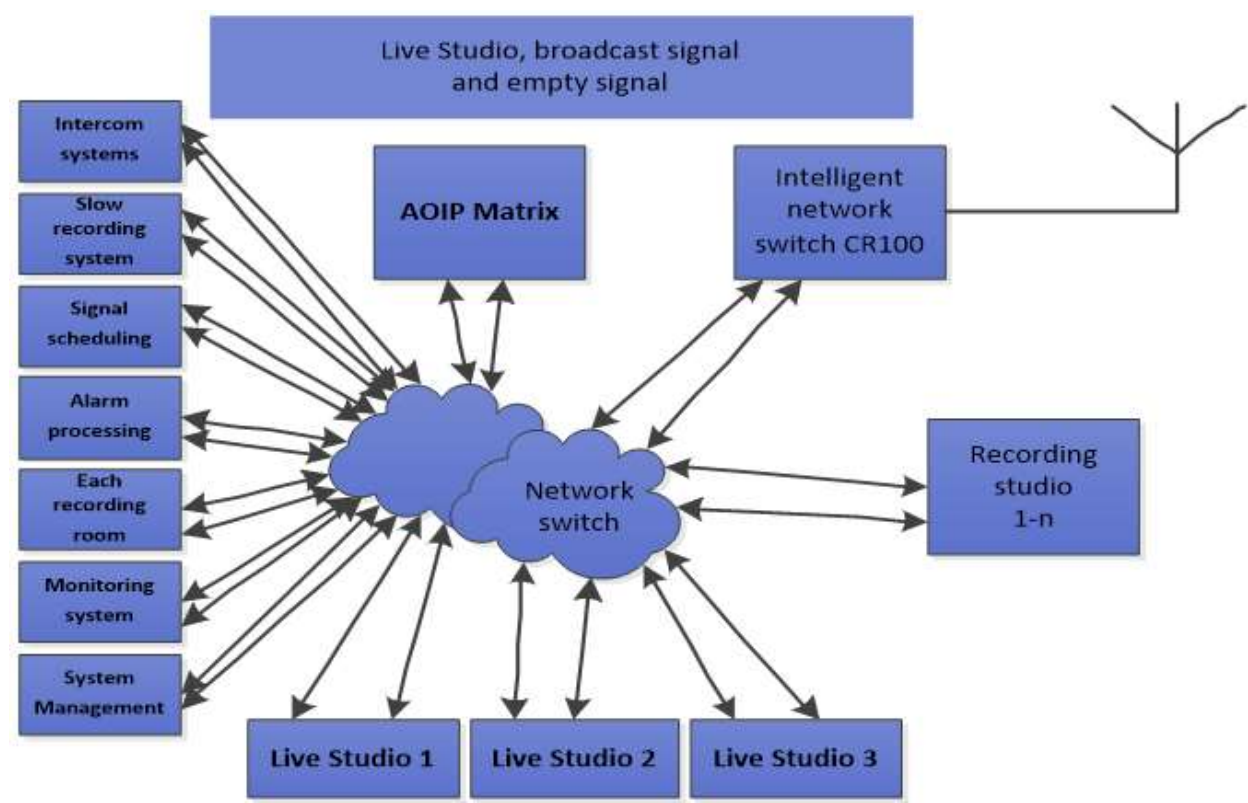

\section{Figure 3. The New Generation of AolP central Control System Platform Structure Diagram}

As is shown in the diagram, the core of the new generation of AoIP central control system is the LAN architecture AoIP system platform. The system is a double network structure, which uses TCP/IP network protocol to real-timely transmit audio signal in the form of IP packets on the LAN or WAN. Each packet has a unique IP address, through which IP addresses can be allocated in the network routing. Control signals and audio signals, video surveillance, and audio collection transmit together in IP way, sharing a network. 


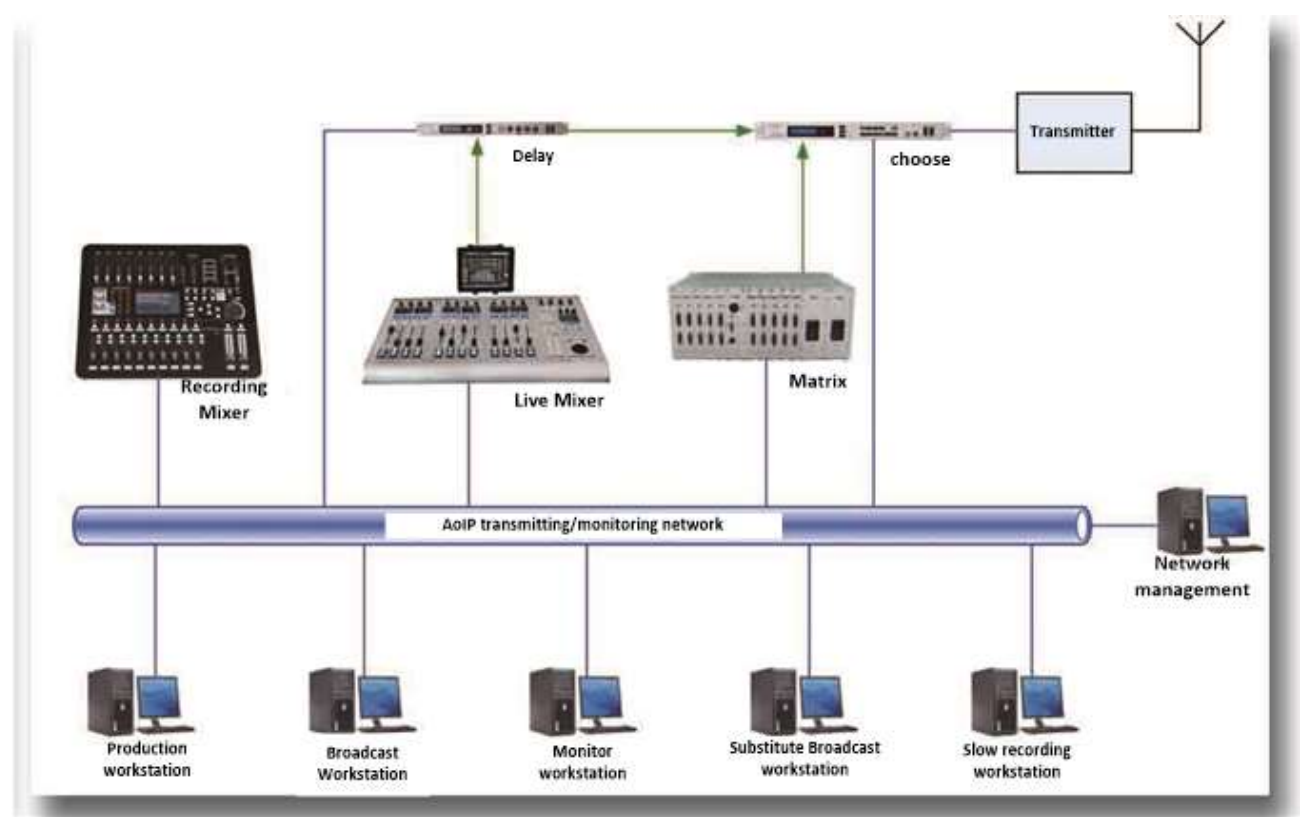

Figure 4.The New Generation of AolP central Control System Connection Diagram

Main technology and function features of this system:

It can use the existing standard of IP network, connect mixer, matrix, audio, routers and other equipment which support AOIP protocol, complete audio scheduling and transmission based on network, and make the system structure more simple, flexible and convenient;

Completing audio stream routing and equipment management in a single network, It doesn't need to set an independent private network like CobraNet. A primary and a standby cable can achieve all access devices and control function, which can make reliability higher and failure rate lower, and be conducive to build a unified and integrated management and scheduling platform;

Workstation is directly connected to the AoIP network by using the virtual sound card, eliminating the intermediate conversion of the AoIP-AES3/ simulation, sound card and workstation. And it is suitable for the replacing of broadcasting, monitoring, slow recording, monitoring, displaying and so on;

AoIP network can bring together different nodes of the audio signal, and implement content-based analysis and monitoring. Through the relevant algorithms to obtain real information such as content relevance, noise, frequency response, and eliminated wave. AOIP network avoids the pseudo monitoring phenomenon caused by analyzing after simplifying signal into a level table like the past;

Easily build digital audio synchronous transmission network of digital audio network by AoIP clock synchronization feature;

Use AoIP's mass transferring characteristics to realize the true meaning of digital audio cloud platform. 


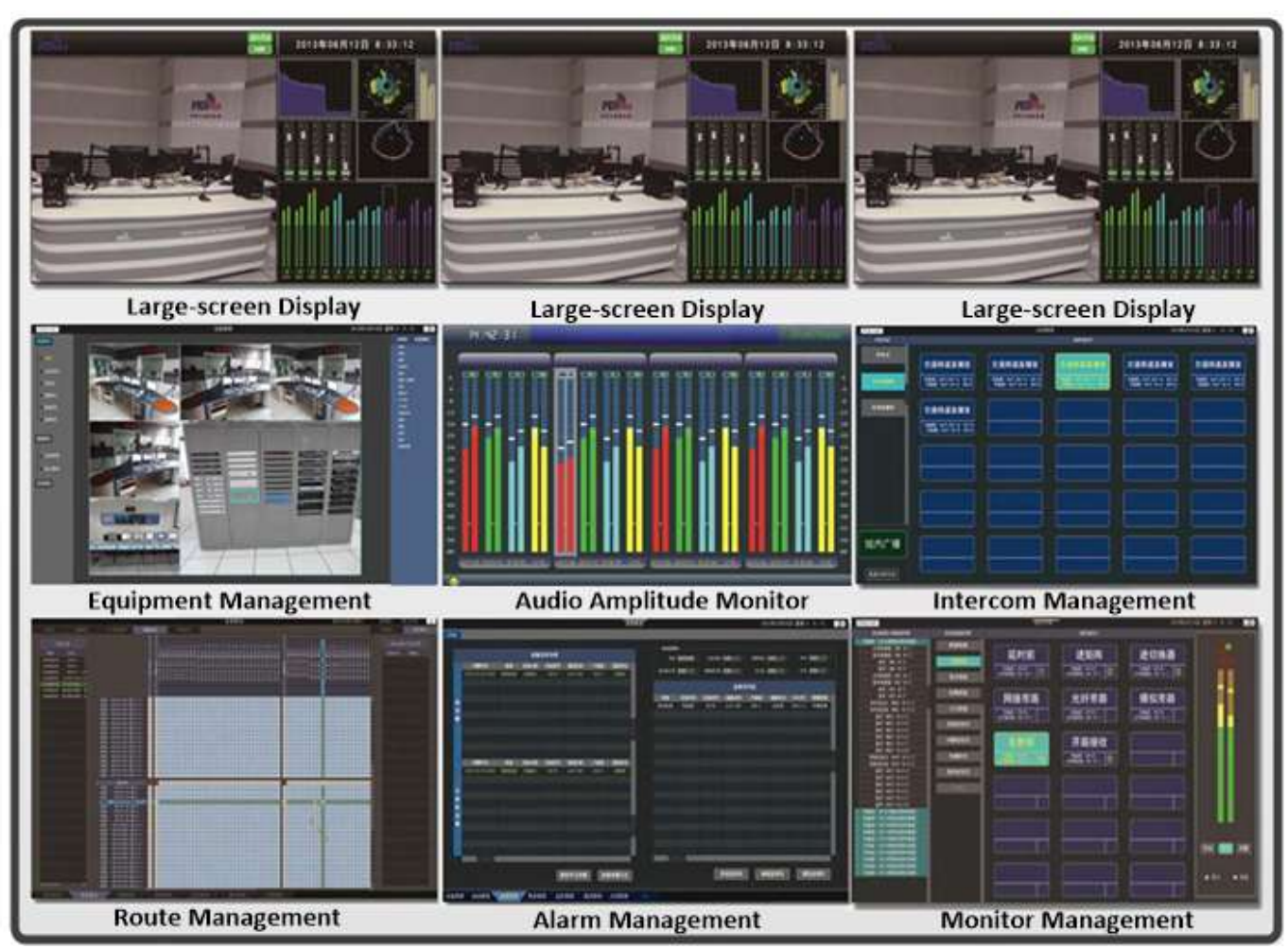

Figure 5. Based on the AoIP Central Control Management Module

\subsection{Introduction of the Key Equipment}

Software module based on AoIP central control system management includes Large-screen display, equipment management, routing management, alarm management, monitoring management, intercom, etc., which cover the audio data, audio parameters, user information of righteousness, monitoring image, the acquisition of environment parameter. It provides sound and light alarm to make corresponding processing plan, has the perfect $\log$ for later analysis and statistics, and also has equipment management, succession management, transmission scheduling management, and other daily information management functions. The module realizes recombinant display, real-timely monitoring, analysis and routing in a graphical way by the frequency in each display terminal.

\section{Conclusion}

AoIP digital audio transmission technology is proposed by Audinate in 2003 based on the Ethernet layer 3 high fidelity real-timely multi-channel audio transmission technologies. Compared with the previous conventional audio transmission technology, it not only inherits all the advantages of CobraNet and EtherSound, but also has its own unique advantages. Our system is designed to provide a stable and reliable new generation of central control system for all levels of radio stations. The application of this technology will bring a new technological revolution to the central control system of Broadcast Network. 


\section{Acknowledgements}

This work was supported by the Institute of Automation, UESTC, and the authors would like to acknowledge its generous donations.

\section{References}

[1] M. Waeltermann, B. Lewcio, P. Vidales and S. Moller, "A technique for seamless VoIP-codec switching in Next Generation Networks", Proceedings of the IEEE International Conference on Communications (ICC 2008), Beijing, China, (2008).

[2] M. Graubner, P. S. Mogre, R. Steinmetz and T. Lorenzen, "A new QoE model and evaluation method for broadcast audio contribution over IP". Proceedings of the 20th ACM Workshop on Network and Operating System Support for Digital Audio and Video, Amsterdam, Netherlands, (2010).

[3] L. Roychoudhuri, E. AI-Shaer and G. B. Brewster, "On the impact of loss and delay variation on Internet packet audio transmission". Computer Communications, vol. 29, no. 10, (2006), pp. 1578-1589.

[4] T. B. Reddy, J. P. John and C. S. R. Murthy, "Providing MAC QoS for multimedia traffic in 802.11e based multi-hop ad hoc wireless networks", Computer Networks, vol. 51, no. 1, (2007), pp. 153-176.

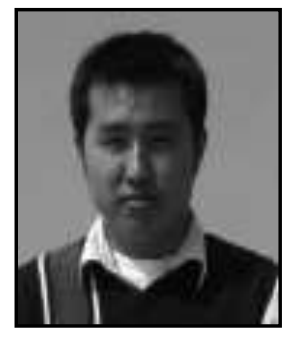

\section{Authors}

Jian He, he is a Lecturer of the University of Electronic Science and Technology of China (UESTC). He got the Master's degree in 2003 at the school of Automation Engineer of the UESTC. And now major in Artificial intelligence, Speech recognition, and the radio and television auto control technology.

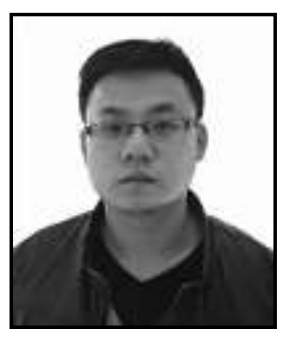

Zhao Zhang, he is a postgraduate student of the University of Electronic Science and Technology of China (UESTC), majoring in Control science and Engineering. 
International Journal of Control and Automation

Vol. 10, No. 6 (2017) 